\title{
Estimación de biomasa aérea y carbono, en rodales con y sin manejo forestal en la Reserva de la Biosfera Mariposa Monarca
}

\section{Aboveground biomass and carbon estimation in stands with and without forest management at the Monarch Butterfly Biosphere Reserve}

\author{
José Antonio Hernández-Moreno', Alejandro Velázquez-Martínez", Aurelio Manuel Fierros-González', Armando \\ Gómez-Guerrero', Valentín José Reyes-Hernández’ y José Amando Gil Vera-Castillo²
}

1 Colegio de Postgraduados. Campus Montecillo. Postgrado Forestal. Estado de México, México.
2 Universidad Autónoma Chapingo. División de
Ciencias Forestales. Texcoco, Estado de México, México.
* Autor de correspondencia.alejvela@colpos.mx

\section{RESUMEN}

La estimación de biomasa aérea acumulada en un bosque con manejo para el aprovechamiento forestal maderable es de importancia para evaluar la sustentabilidad por efecto del sistema silvícola, y para entender la dinámica y funcionamiento de los ecosistemas forestales. La acumulación de biomasa aérea refleja la productividad en un sitio determinado. En este trabajo se estimó la biomasa aérea y el carbono, en rodales con y sin manejo forestal, en el Ejido Chincua, Senguio, Michoacán, a través de la generación de ecuaciones alométricas para Abies religiosa (Kunth) Schltdl. \& Cham y Pinus pseudostrobus Lindl, mientras que para las latifoliadas se usaron ecuaciones de otros estudios y, además, mediante datos de inventario para todas las especies. El rodal sin manejo tuvo una biomasa de $323.23 \mathrm{Mg} \mathrm{ha}^{-1}$, mientras que el rodal con manejo $287.62 \mathrm{Mg} \mathrm{ha}^{-1}$, los cuales, a pesar de su variabilidad no presentan diferencias significativas $(\mathrm{P}=0.96)$. El contenido de carbono tampoco presentó diferencias significativas $(\mathrm{P}=0.97)$, siendo $155.03 \mathrm{Mg}$ ha ${ }^{-1} \mathrm{y} 128.44 \mathrm{Mg}$ $\mathrm{ha}^{-1}$ en los rodales sin y con manejo, respectivamente. Los resultados indican que con el manejo forestal, bajo el sistema silvícola de selección, se conservaron la estructura y composición del rodal con manejo, lo que permitió mantener el almacén de biomasa en una cantidad similar a la de un bosque sin manejo; por lo anterior, los bosques manejados pueden ser sumideros eficientes de carbono, más la cantidad de este elemento en el proceso de cosecha y transformación de la madera a productos forestales de larga duración, como son madera para construcción, muebles, durmientes, postes, entre otros, que permiten su permanencia por periodos relativamente largos.

Palabras Clave: Abies religiosa; cambio climático; ecuaciones alométricas; Pinus pseudostrobus; Sierra Chincua; sistema silvícola de selección.

\section{ABSTRACT}

The estimation of accumulated aboveground biomass in a forest with management for timber harvesting is of importance to evaluate sustainability regarding the effect of the silvicultural system, and to understand the dynamics and functioning of forest ecosystems. Aboveground biomass accumulation reflects productivity at a given site. In this work we estimated the aboveground biomass and the carbon, in stands with and without forest management, in the Chincua, Senguio, Michoacán Ejido, through the generation of allometric equations for Abies religiosa (Kunth) Schltdl. \& Cham and Pinus pseudostrobus Lindl, while for the broadleaf equations from other studies were used and, in addition, by means of inventory data for all species. The stand without management had a biomass of $323.23 \mathrm{Mg}$ ha ${ }^{-1}$, while the stand with management $287.62 \mathrm{Mg} \mathrm{ha}^{-1}$, which, despite its variability, did not present significant differences $(\mathrm{P}=0.96)$. The carbon content also did not present significant differences $(\mathrm{P}=0.97)$, being $155.03 \mathrm{Mg} \mathrm{ha}^{-1}$ and $128.44 \mathrm{Mg}^{-1}$ in the stands without and with management, respectively. The results indicate that with forest management, under the silvicultural selection system, the structure and composition of the managed stand were conserved, which allowed maintaining the biomass storage in an amount similar to that of a forest without management; Therefore, managed forests can be efficient carbon sinks, plus the amount of this element in the process of harvesting and processing of wood to long-term forest products, such as construction timber, furniture, railways ties, utility poles, among others, which allow their permanence for relatively long periods.

KEYWORDS: Abies religiosa; climate change; allometric models; Pinus pseudostrobus; Sierra Chincua; silvicultural selection system. 


\section{INTRODUCCIÓN}

Los niveles atmosféricos de $\mathrm{CO}_{2}$ han aumentado drásticamente en las últimas cinco décadas de 314.62 ppm (1958) a 411 ppm (Administración Nacional de la Aeronáutica y del Espacio [NASA], 2018). Este aumento se ha convertido en un problema a escala mundial y plantea desafíos enormes para el clima, los bosques y la humanidad, obligando a la búsqueda de estrategias para mitigar sus impactos. Las comunidades vegetales arbóreas son el principal almacén terrestre de biomasa aérea (Perry et al., 2008; Rackley, 2010; Galicia et al., 2015) debido a que, mediante la fotosíntesis, absorben cantidades importantes de $\mathrm{CO}_{2}$ atmosférico, fijando carbono en sus tejidos y transfiriéndolo al suelo mediante el proceso de descomposición de materia orgánica. Lo anterior se refleja globalmente en el inventario de carbono total de la biosfera terrestre, estimado en 2200 Gt (Ehlers y Krafft, 2001), contenido tanto en la biomasa viva (hojas, ramas, tallos y raíces), representando $27 \%$, como en los suelos (hojarasca y materia orgánica del suelo), siendo el restante $73 \%$ (Rackley, 2010). Los bosques y selvas retienen altas densidades de carbono (masa por unidad de área), en comparación con otros tipos de vegetación (Perry et al., 2008). La biomasa aérea representa más de $40 \%$ del carbono total almacenado en los bosques a nivel mundial (Rackley, 2010; Galicia et al., 2015; González de Tanago et al., 2017). Factores como la edad, la densidad y la composición florística de cada estrato por comunidad vegetal, modifican directamente la capacidad de los ecosistemas forestales para almacenar carbono (Schulze $e t$ al., 2000), estos factores generalmente se modifican con el manejo forestal y se reflejan en cambios en la distribución de carbono dentro del ecosistema.

En las últimas décadas, se han realizado estudios para estimar la biomasa aérea de árboles individuales y masas forestales como los publicados para diferentes especies y ecosistemas forestales (Acosta-Mireles et al., 2002; Návar, 2010; Acosta et al., 2011; Aguilar et al., 2012; Rojas-García et al., 2015; Aguilar-Hernández et al., 2016). Sin embargo, los estudios en bosques con manejo para el aprove- chamiento forestal maderable son escasos, y más aún, en las zonas de conservación, que forman parte de áreas naturales protegidas (ANP) en México, siendo estas últimas, una de las estrategias de política ambiental para frenar los procesos de deterioro ambiental, para conservar la diversidad biológica y para fomentar el uso sustentable de los recursos naturales (Comisión Nacional de Áreas Naturales Protegidas [Conanp], 2001). Por lo anterior, surgió el interés de estimar los reservorios y el potencial de almacenamiento de biomasa y carbono en el área bajo estudio, aunado al fenómeno de migración de la mariposa monarca (Danaus plexippus), desde los bosques del sur de Canadá hasta los bosques de la Reserva de la Biosfera Mariposa Monarca (RBMM) en Michoacán y el Estado de México. Es necesario destacar que la RBMM enfrenta un serio problema respecto a la abundancia y calidad de sus bosques (Conanp, 2001), afectando algunos procesos y funciones ecosistémicos, a la flora y fauna que los habita, así como a las personas que de esta dependen, ya que los bosques de la región prestan distintos tipos de servicios ecosistémicos, como son: conservación de suelo, captura de carbono, captación de agua y recarga de acuíferos, producción de madera, entre otros. Los ecosistemas más vulnerables al cambio climático podrían ser los bosques de pino y oyamel que crecen a una altitud superior a los 2800 m snm (Dirzo, 2001), que es en donde se encuentra el ANP en estudio. En este contexto, el aprovechamiento forestal sostenible se considera una estrategia para la adaptación y mitigación contra el incremento de $\mathrm{CO}_{2}$ atmosférico (Galicia et al., 2015). El papel de la silvicultura y el manejo forestal, que tienen como objetivo la producción maderable, es promover la conservación de los ecosistemas forestales, la producción de bienes y servicios para la sociedad, y al mismo tiempo, reducir la deforestación y degradación; lo cual, en su conjunto, se considera como una estrategia para fijar carbono y reducir las emisiones de $\mathrm{CO}_{2}$.

\section{OBJETIVOS}

El objetivo del estudio fue estimar la biomasa aérea total (BA) y contenido de carbono (CC) en rodales mixtos con y sin manejo, bajo la hipótesis de que, de acuerdo con el 
historial de manejo, no existen diferencias significativas entre el bosque con manejo y sin manejo en cuanto al almacenamiento total de biomasa y carbono de la parte aérea, y así explicar la importancia del aprovechamiento forestal sustentable en bosques de las ANP, su función en el almacenamiento de carbono atmosférico y sus beneficios, de manera cuantitativa.

\section{MATERIALES Y MÉTODOS}

\section{Área de estudio}

La RBMM se localiza entre los estados de México y Michoacán, en los municipios de Temascalcingo, San José del Rincón, Donato Guerra y Villa de Allende, Estado de México, y Contepec, Senguio, Angangueo, Ocampo,

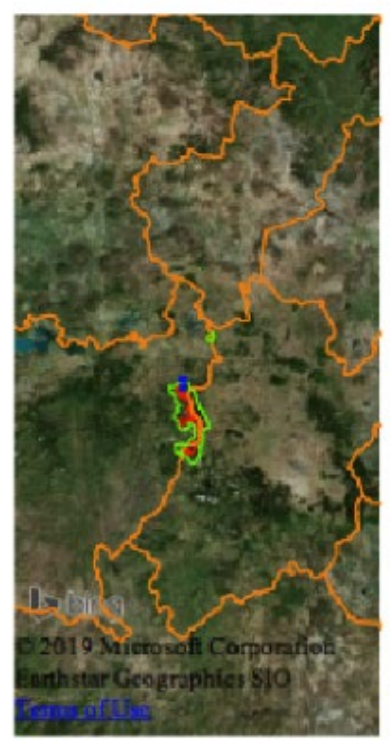

ESCALA 1:3,500,000

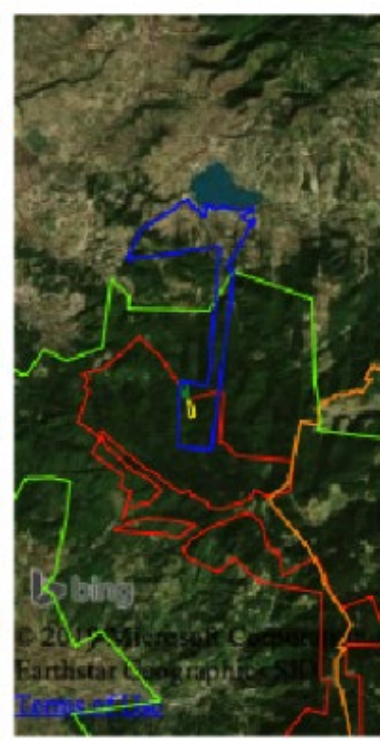

ESCALA 1:200,000
RESERVA DE LA BIOSFERA MARIPOSA MONARCA

\section{EJIDO CHINCHUA, MPIO. SENGUIO, MICHOACÁN}

LOCALIZACIÓN DEL ÁREA DE ESTUDIO

Proyección WGS84 UTM; Elaboradi a partir de datos INEGI, ESRI, BING
Zitácuaro y Áporo, Michoacán (Fig. 1). Sus coordenadas geográficas extremas son: $19^{\circ} 44^{\prime} 27^{\prime \prime}$ y $19^{\circ} 18^{\prime} 32^{\prime \prime}$ de latitud N y $100^{\circ} 22^{\prime} 26^{\prime \prime}$ y $100^{\circ} 09^{\prime} 07^{\prime \prime}$ de longitud O. Tiene una superficie de 56259.05 ha, divididas en tres zonas núcleo: "Altamirano", "Sierra Chincua-CampanarioChivatí-Huacal" y "Cerro Pelón”, que abarcan una superficie de 13551.55 ha y dos zonas de amortiguamiento de 42707.49 ha (Conanp, 2001).

El presente estudio se llevó a cabo en el ejido Chincua, municipio de Senguio, al oriente del estado de Michoacán, y se localiza entre los $19^{\circ} 44^{\prime} 24.45^{\prime \prime}$ y $19^{\circ} 41^{\prime} 16.17^{\prime \prime}$ de latitud $\mathrm{N}, \mathrm{y} \operatorname{los} 100^{\circ} 17^{\prime} 25.18^{\prime \prime}$ y $100^{\circ} 17^{\prime} 52.21^{\prime \prime}$ de longitud $\mathrm{O}$, forma parte de la zona núcleo y zona de amortiguamiento del "Santuario Sierra ChincuaCampanario-Chivatí-Huacal”, al norte de la RBMM (Fig. 1).

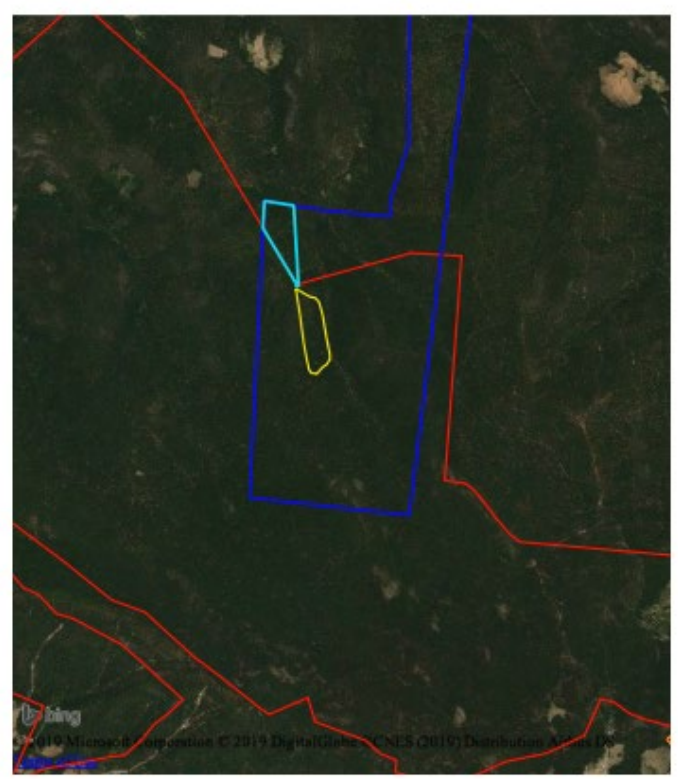

ESCALA 1:40,000

Leyenda

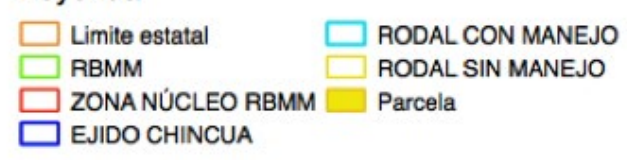

FIGURA 1. Localización de la RBMM y del área de estudio. 
Se ubica en la provincia fisiográfica del Eje Neovolcánico, subprovincia Mil Cumbres. El relieve está formado por un sistema de topoformas constituidas por sierras volcánicas complejas, con altitudes que varían desde los $2500 \mathrm{~m}$ hasta los $3600 \mathrm{~m}$ snm, con pendientes de entre $10 \%$ y $50 \%$, y la exposición que domina es norte y noroeste. De acuerdo con cartas edafológicas del Instituto Nacional de Estadística y Geografía [Inegi] (2014), los suelos son derivados de cenizas volcánicas y los principales tipos, de acuerdo con la Base Referencial Mundial del Recurso Suelo (WRB, 2015) son: Andosol húmico y Acrisol hórtico, con textura media. El clima es templado subhúmedo (C (w2)), con temperatura media anual entre $8{ }^{\circ} \mathrm{C}$ y $22^{\circ} \mathrm{C}$. La precipitación total anual oscila entre $700 \mathrm{~mm}$ y $1250 \mathrm{~mm}$, con lluvias en verano, que comprenden entre 20\% y 35\% de la total anual (García, 2004). Los principales tipos de vegetación a nivel regional son: bosque de pino-encino, bosque de cedro y bosque de oyamel (Inegi, 2016).

El estudio se realizó en dos condiciones: en un rodal de 9.42 ha de superficie con manejo forestal (RCM) bajo el sistema silvícola de cortas de selección, ubicado en la zona de amortiguamiento de la RBMM (de acuerdo con el programa de manejo de la RBMM, está permitido el aprovechamiento forestal sustentable bajo la regulación y control estrictos de acuerdo con la normatividad en materia ambiental de la Secretaría), y en un rodal sin manejo forestal o conservado (RSM) de 9.27 ha, dentro de la zona núcleo, donde el aprovechamiento forestal está prohibido (Fig. 1). Como se puede ver en la figura 1 , ambos rodales se encuentran en la zona limítrofe de amortiguamiento y núcleo de la RBMM (Conanp, 2001), y presentan un dosel cerrado de pino (Pinus pseudostrobus Lindl.), oyamel (Abies religiosa (Kunth) Schltdl. \& Cham.), encinos (Quercus laurina Humb. \& Bonpl. y Quercus rugosa Née) y otras especies arbóreas latifoliadas de talla menor como el Alnus acuminata Kunth subsp. glabrata (Fernald) Furlow, Arbutus xalapensis Kunth, Clethra mexicana DC.

Los rodales comparten especies arbóreas, arbustivas y herbáceas, a excepción de Clethra mexicana, que solo se encuentra en el RSM. Los rodales son contiguos y de condiciones similares en estructura y composición, expo- sición noreste, altitud media de $2925 \mathrm{~m}$ snm, topografía y tipo de suelo similares, y no se han registrado incendios por lo menos en los últimos 20 años (Fig. 1).

\section{Sitios de muestreo y medición de variables dasométricas}

El ejido posee una superficie bajo manejo forestal maderable de 343.1 ha, dividida en 10 áreas de corta. El RCM se cosechó con el sistema silvícola de selección individual y en grupos en los años 1997 (690 m³ VTA) y 2007 (711 m³ VTA) y corresponde al área de corta 10, según el programa de manejo forestal maderable (PMFM) del ejido Chincua; mientras que el RSM se ha conservado bajo condiciones naturales, sin corta y tiene una edad aproximada de 90 años, de acuerdo con los programas de manejo de la RBMM y el del ejido Chincua (Conanp, 2001). En cada rodal se establecieron 6 sitios de muestreo circulares de 0.1 ha en el 2017, a lo largo de un transecto con rumbo sureste y con un intervalo altitudinal de los 2885 m a los 2965 m snm (Fig. 1), midiendo todos los árboles con $\mathrm{DN} \geq 7.5 \mathrm{~cm}$. La información se complementó con datos de un inventario realizado en el año 2007 para ambos rodales, los cuales se utilizaron para comparar y evaluar el contenido de biomasa aérea del bosque en dos años diferentes (2007 y 2017).

Se calculó en índice de valor de importancia (IVI), para cada condición (RCM y RSM), mediante la suma de los parámetros de la estructura horizontal (abundancia, frecuencia y dominancia), para las especies del estrato arbóreo (Curtis y McIntosh, 1951).

\section{Estimación de la biomasa aérea total (BA)}

La BA se estimó mediante modelos generados en el presente estudio para coníferas, y a través de modelos alométricos generados en otros estudios para latifoliadas (Acosta-Mireles et al., 2002; Návar, 2010; Acosta et al., 2011; Aguilar et al., 2012; Rojas-García et al., 2015; AguilarHernández et al., 2016). Se derribaron 12 árboles para generar ecuaciones alométricas, seis de Pinus pseudostrobus y seis de Abies religiosa. La selección de árboles para su derribo 
estuvo en función de la disponibilidad dentro del área de corta, con fuste recto y libre de daños, de dimensiones representativas de las categorías diamétricas, esto en la medida de lo posible, ya que el diámetro mínimo de corta en los aprovechamientos forestales para la región es de 30 cm de DN, como lo marca el PMFM. En cada árbol seleccionado se midió el DN con cinta diamétrica, antes de ser derribado, y posteriormente se separaron tres componentes estructurales: fuste, ramas y follaje. La metodología usada fue la descrita por Picard et al. (2012), y es similar a la que han empleado en estudios similares (Acosta-Mireles et al., 2002; Díaz et al., 2007). El fuste se seccionó a $2.55 \mathrm{~m}$ y $1.25 \mathrm{~m}$ de longitud, medidas comerciales maderables, y se obtuvo una rodaja de aproximadamente $5 \mathrm{~cm}$ de espesor de cada troza (Fig. 2), el número de muestras por árbol fue variable, dependiendo de la longitud y diámetro del fuste.

Se tomaron 3 muestras de ramas y 3 de follaje por árbol, de aproximadamente 500 g. Para obtener el peso verde, cada componente del árbol, muestras y rodajas se pesaron in situ, evitando pérdida de humedad, con una báscula digital tipo grúa (TOR-REY SERIE CRS) de 500 $\mathrm{kg}$ (resolución de $0.2 \mathrm{~kg}$ ) para trozas y con una báscula digital (VINSON Rhino) de $40 \mathrm{~kg}$ (resolución de $0.002 \mathrm{~kg}$ ) para las rodajas y muestras. Las muestras se transportaron al laboratorio en donde se secaron en una estufa de circulación forzada a $70{ }^{\circ} \mathrm{C}$, hasta obtener el peso seco constante. Con el peso seco así calculado, se determinó la relación peso seco/peso fresco de rodajas, ramas y follaje, y estos coeficientes se multiplicaron por su respectivo peso fresco para determinar la biomasa de cada componente.

La BA por árbol se determinó con la suma de la biomasa del fuste, ramas y follaje. Una vez obtenidos los datos de DN y BA por árbol, se seleccionó un modelo potencial por ser uno de los más frecuentemente usados, debido a que proporciona buenos ajustes (Acosta-Mireles et al., 2002; Díaz et al., 2007; Acosta-Mireles et al, 2009; Avendaño et al., 2009; Rodríguez-Laguna et al., 2009); TerMikaelian y Korzukhin, 1997)

$$
Y=b X^{k}
$$

donde:

$Y$ es la biomasa o carbono $(\mathrm{kg})$

$X$ es el diámetro normal ( $\mathrm{m})$

by k son los parámetros estimados

El área basal por rodal también incluyó otras especies de latifoliadas (Quercus laurina Humb et Bonpl, Q. rugosa Neé, Alnus acuminata subsp. Glabrata H.B.K., Arbutus xalapensis Kunth y Clethra mexicana A. DC) presentes al momento de realizar el inventario. Las ecuaciones para estimarla se muestran en la tabla 1. Los valores de BA se estimaron aplicando las ecuaciones alométricas a los árboles individuales de los datos de inventario, dentro de los sitios de muestreo, sumándolos y dividiendo entre la superficie de los sitios para obtener valores en megagramos por hectárea.

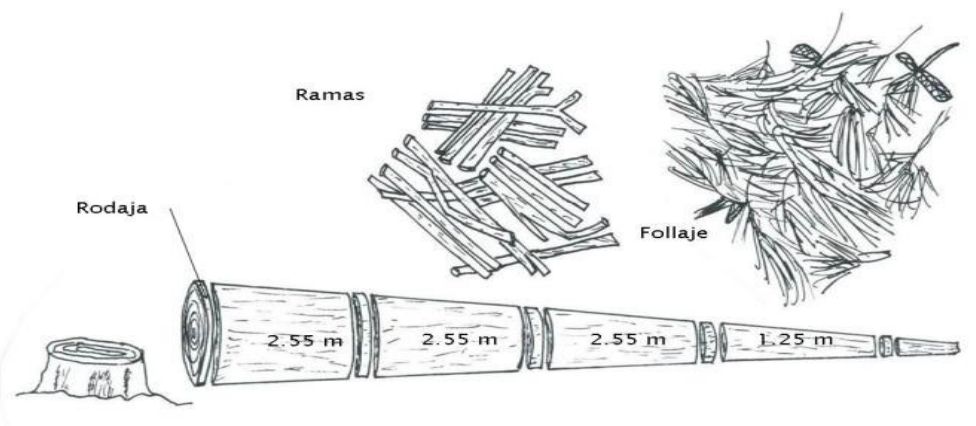

FIGURA 2. Troceo del arbolado, obtención de rodajas y separación por componente estructural (Tomado de Díaz et al., 2007). 
TABLA 1. Ecuaciones alométricas para estimar la biomasa aérea en especies latifoliadas.

\begin{tabular}{lllc}
\hline Especie & Ecuación & $R^{2}$ & Referencia \\
\hline $\begin{array}{l}\text { Quercus laurina } \\
\text { Quercus rugosa }\end{array}$ & $\mathrm{BA}=$ EXP[-4.3375+1.0760(DN2xAT)] & 0.96 & Ruiz-Aquino et al. (2014) \\
$\begin{array}{l}\text { Alnus acuminata subsp. } \\
\text { glabrata }\end{array}$ & $\mathrm{BA}=0.0342 \mathrm{DN}^{2.759}$ & 0.92 & Aguilar et al. (2012) \\
$\begin{array}{l}\text { Arbutus xalapensis } \\
\text { Clethra mexicana }\end{array}$ & $\mathrm{BA}=\left(0.3764 \mathrm{DN}^{-(-2.4)} \mathrm{DN}^{2.23}-(2.3146 \mathrm{DN})-(1.9106)\right.$ & 0.99 & Acosta-Mireles et al. (2002) \\
\hline BA = Biomasa aérea total; DN = diámetro normal (m) & $\mathrm{BA}=0.4632 \mathrm{DN}^{1.8168}$ & 0.95 & Acosta et al. (2O11)
\end{tabular}

\section{Determinación del contenido de carbono}

El contenido de carbono (CC) en la biomasa de cada árbol se estimó con la expresión (Acosta et al., 2011):

$$
\mathrm{CC}=\mathrm{BxFC}
$$

donde:

CC es el contenido de carbono en el componente

$\mathrm{B}$ es la biomasa de cada árbol $(\mathrm{kg})$

FC es el factor o porcentaje de carbono (\% C) contenido en cada componente

Dentro de la composición elemental de la madera y, en general de la materia vegetal seca, el contenido de carbono representa aproximadamente $50 \%$ del total en tejidos vegetales (MacDicken, 1997; Husch, 2001; Intergovernmental Panel on Climate Change [IPCC], 2001) y, debido a que el porcentaje de carbono contenido en los árboles no varía significativamente entre componentes estructurales, ni de una región a otra (Acosta-Mireles et al., 2009; Rodríguez-Laguna et al., 2009; Carrillo et al., 2014; Hernández, 2015), se utilizaron los datos encontrados en la literatura en estudios previos, para las 7 especies de la presente investigación (Acosta-Mireles et al., 2009; Avendaño et al., 2009; Návar, 2010; Acosta et al., 2011; Rojas-García et al., 2015; Jiménez et al., 2013; Carrillo et al., 2014; Hernández, 2015; Aguilar-Hernández et al., 2016; Díaz-Ríos, 2016 ), usando el promedio de los componentes estructurales (Tabla 2). Para la determinación de la ecuación de carbono, se empleó el mismo modelo que se usó para estimar BA, relacionando el CC y el diámetro normal de cada árbol (Acosta et al., 2011).

\section{Análisis estadístico y procesamiento de datos}

Para generar las ecuaciones de BA, se ajustó el modelo alométrico $Y=b X^{k}$ (Ter-Mikaelian y Korzukhin, 1997), mediante regresión no lineal. Se linealizó el mismo modelo, para facilitar la interpretación del análisis de regresión (Little y Jackson, 1976). El modelo transformado logarítmicamente fue el siguiente: $\ln (Y)=\ln (b)+k \ln (X)$; donde $Y, X, b$ y $k$ tienen el mismo significado que en el modelo no lineal, y $\ln (Y), \ln (b) y \ln (x)$ son los logaritmos naturales de $Y$, by $X$, respectivamente (Acosta-Mireles et al., 2009; Avendaño et al., 2009).

La comparación a priori de BA entre rodales se realizó aplicando un Análisis de Varianza (ANDEVA) de una vía (completamente aleatorio), usando como factor principal el rodal. Antes del ANDEVA, los datos de BA fueron transformados mediante la distribución Johnson para cumplir el supuesto de normalidad de los datos (Slifker y Shapiro, 1980), misma que se verificó con la prueba de Shapiro-Wilk (Shapiro y Wilk, 1965). Se comprobó la homogeneidad de varianzas mediante las pruebas Bartlett, Levene y Fligner-Killeen (Correa et al., 2006). 
TABLA 2. Porcentaje de carbono FC $(\% \mathrm{C})$ para las 7 especies estudiadas.

\begin{tabular}{lcc}
\hline Especie & $\% C$ & Referencia \\
\hline Pinus pseudostrobus & 0.50 & Jiménez et al. (2013) \\
Abies religiosa & 0.47 & Avendaño et al. (2009); \\
Quercus sp. & 0.49 & Jiménez et al. (2013) \\
Alnus sp. & 0.46 & Díaz-Ríos et al. (2O16); Carrillo et al. (2014) \\
Arbutus xalapensis & 0.51 & Aguilar-Hernández et al. (2016); Jiménez et al., 2013 \\
Clethra mexicana & 0.47 & Acosta et al. (2011) \\
\hline
\end{tabular}

El análisis se realizó como repeticiones o "pseudorepeticiones" (Hurlbert, 1984), utilizando valores de BA de RCM y RSM. Se evaluaron las diferencias entre rodales, no existieron repeticiones para el factor edad, ya que solo se utilizaron los datos de BA para cada rodal, y para cada año de registro (2007 y 2017). Se realizó una prueba a posteriori, para determinar que medias de BA por rodal presentan diferencias, en este caso fue la prueba de Tukey HSD $(\alpha \leq$ 0.005).

Las estimaciones de biomasa aérea con las ecuaciones generadas en el presente estudio fueron comparadas con las de otras ecuaciones halladas en la literatura (Avendaño et al., 2009 y Návar, 2010), mediante regresión lineal, entre valores observados y estimados, asimismo, se realizó una prueba de t de student entre estos valores.

Todos los análisis estadísticos se realizaron con el paquete libre R versión 3.4.3 (R Core Team, 2016), para el sistema operativo Macintosh ${ }^{\circledR}$.

\section{Resultados}

\section{Características dasométricas}

Los rodales estudiados presentaron similitudes en estructura y composición, son incoetáneos, mezclados, donde la mayoría de los individuos se encuentran en las categorías diamétricas iniciales. Como se puede observar en la línea de tendencia de la figura 3, el RCM tiende a la distribución normal, tomando como base la curva normal de distribución de Liocourt (crecimiento tipo "J” invertida).

Se obtuvo que el mayor índice de valor de importancia (IVI), en ambos rodales, lo tuvieron: Q. laurina (RCM = $35 \%$ y RSM $=31.4 \%)$, P. pseudostrobus $(\mathrm{RCM}=24.1 \% \mathrm{y}$ RSM $=25.9 \%$ ). La tercera especie, en orden de IVI, resultó ser A. religiosa (12.3\%) para el RCM, y Q. rugosa (13.4\%) para el RSM. Las otras especies se encontraron en el intervalo de $2.8 \%$ a $12.58 \%$ de IVI (Fig. 4). Ambos rodales presentaron características dasométricas similares en cuanto a número de árboles por hectárea (NAH) y área basal ( $\mathrm{AB})$, las cuales influyen directamente en la acumulación de BA y CC (Tabla 3 ), entre las cuales no existe diferencia significativa ( $\mathrm{P} \leq$ $0.99)$.

\section{Ecuaciones de biomasa y carbono}

Las ecuaciones generadas para BA y CC, presentaron un coeficiente de determinación $\mathrm{R}^{2}=0.96$ para Abies religiosa (Kunth) Schltdl. \& Cham. (Figs. 4a y 5a) y R $\mathrm{R}^{2}=0.93$ en Pinus pseudostrobus Lindl. (Figs. 4b y 5b).

Los modelos, sus parámetros y la dispersión de puntos de los valores observados de BA y CC (kg), se presentan en las figuras 4 y 5 indicando que las ecuaciones alométricas de ambas especies son confiables (Tabla 4). 

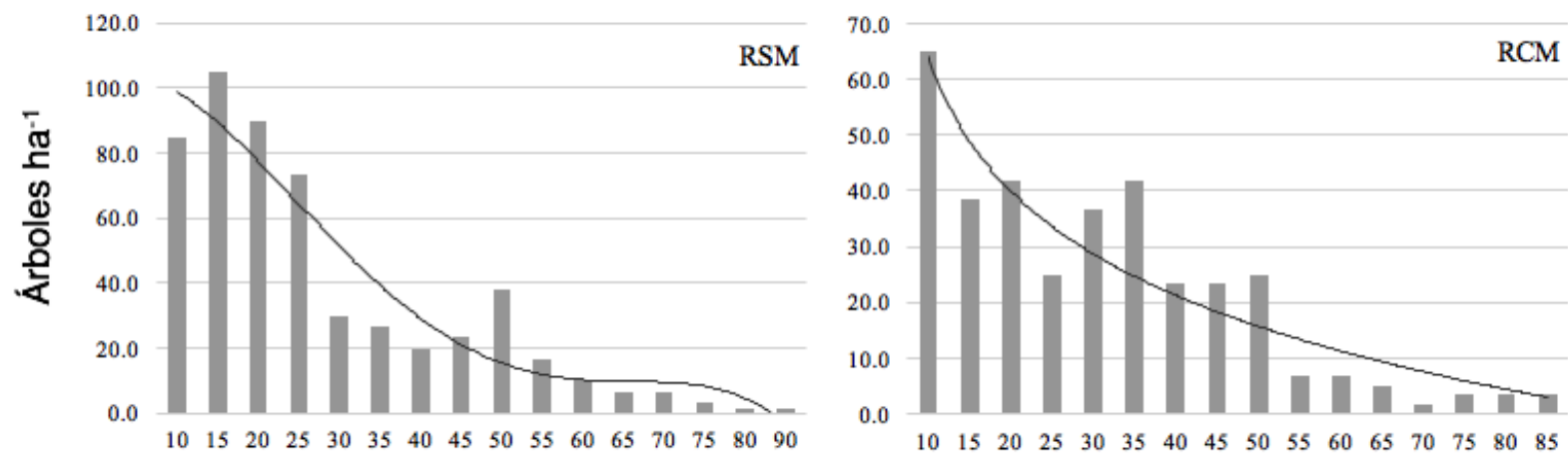

\section{Categoría diamétrica $(\mathrm{cm})$}

FIGURA 3. Frecuencia por categoría diamétrica en RSM (izquierda) y RCM (derecha).

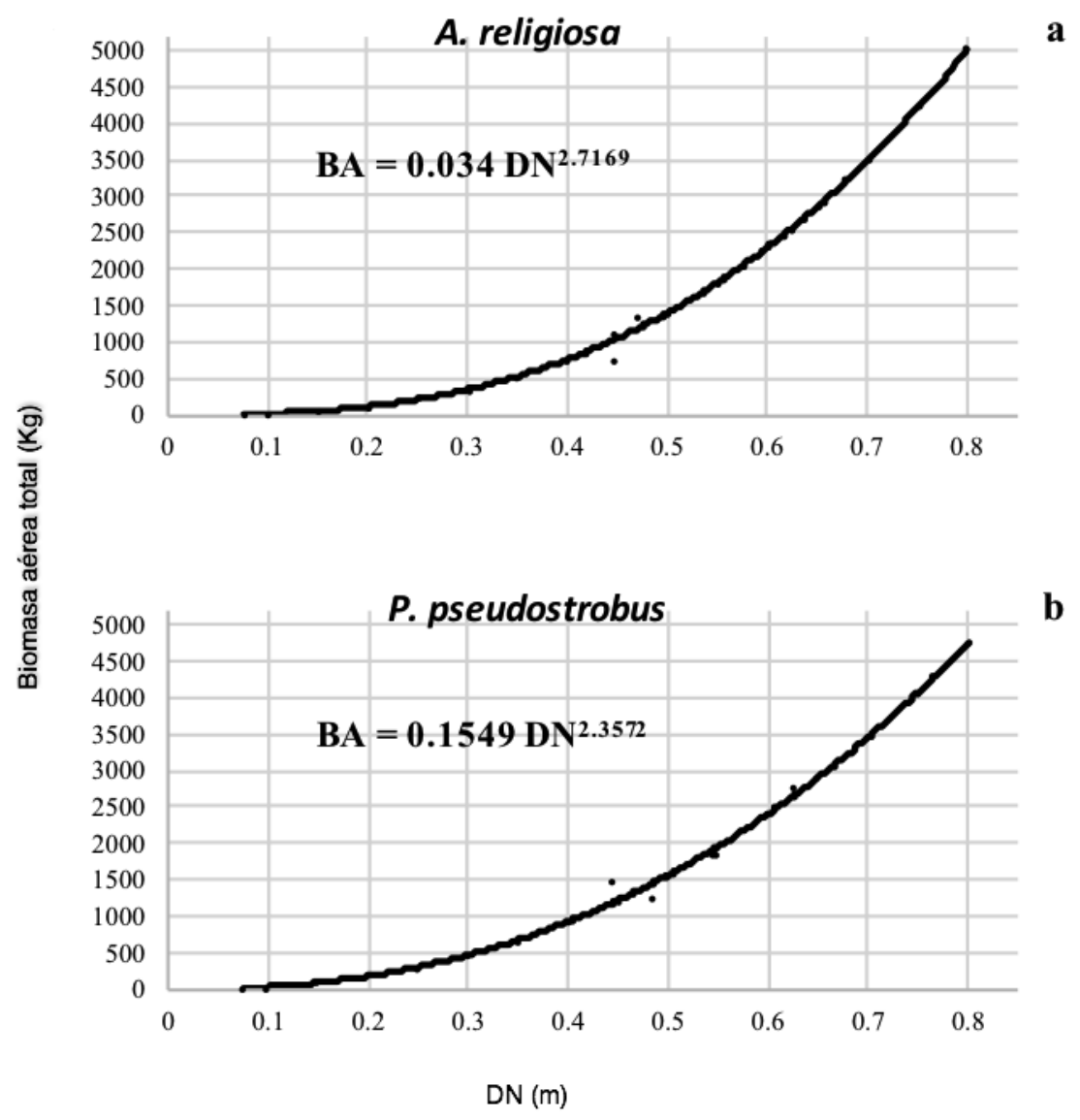

FIGURA 4. Dispersión de valores de biomasa, línea de regresión y ecuación generada, para los modelos potenciales de Abies religiosa (Kunth) Schltdl. \& Cham. (a) y Pinus pseudostrobus Lindl. (b).

Los parámetros de ecuaciones, los errores estándar de estimación, los coeficientes de determinación $\left(R^{2}\right)$ y los valores de p se muestran en la tabla 
TABLA 3. Área basal y densidad en RCM y RSM (2007-2017).

\begin{tabular}{cccc}
\hline Condición del Rodal & Año & Área basal $\left(\mathrm{m}^{2} \mathrm{ha}^{-1}\right)$ & Densidad (arboles $\left.\mathrm{ha}^{-1}\right)$ \\
\hline RCM & 2007 & 29.76 & 334.78 \\
RSM & 2007 & 44.11 & 354.79 \\
RCM & 2017 & 32.63 & 350.00 \\
RSM & 2017 & 42.52 & 438.33 \\
\hline
\end{tabular}

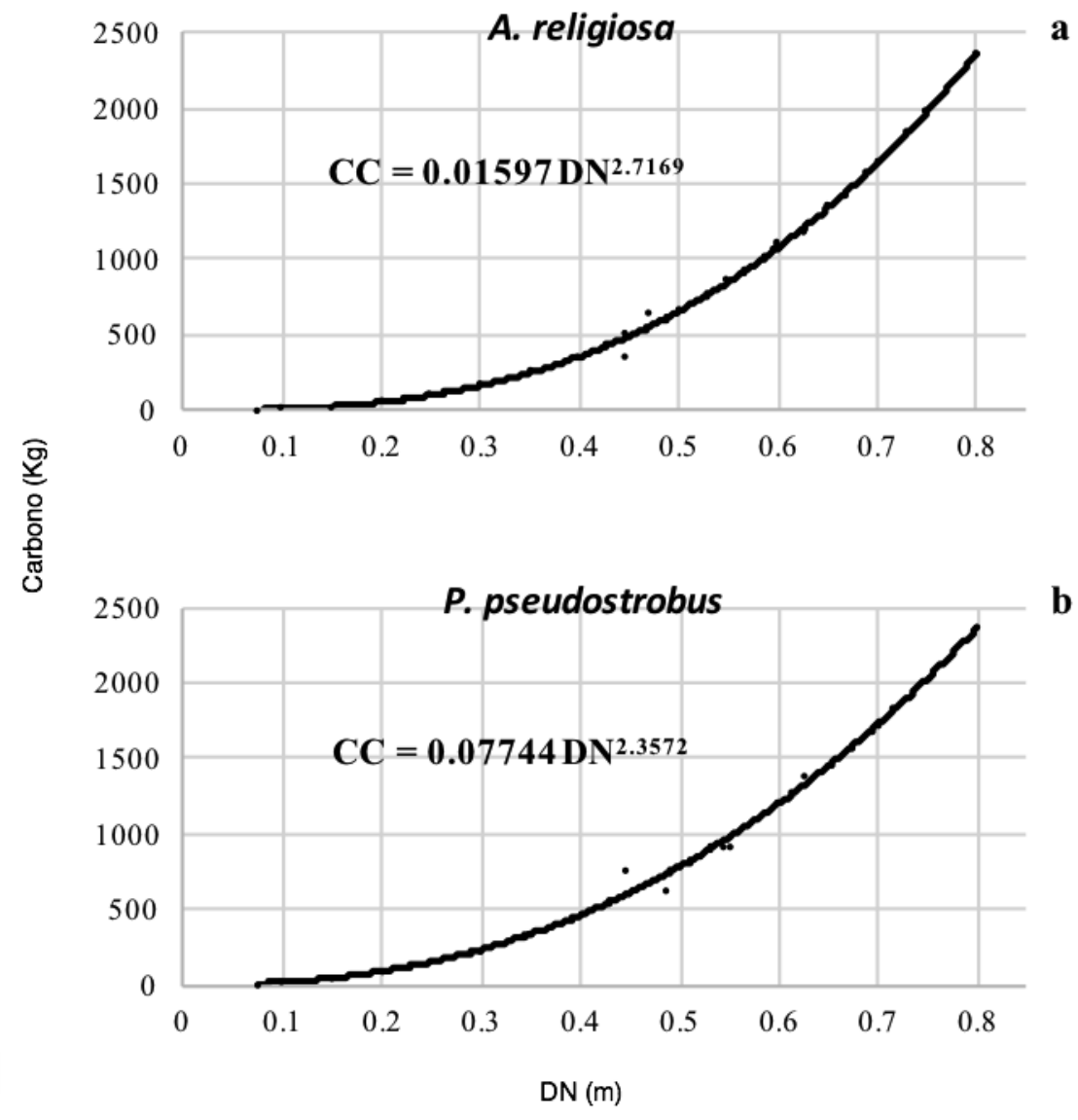

FIGURA 5. Dispersión de valores de carbono, línea de regresión y ecuación generada, para los modelos potenciales de Abies religiosa (Kunth) Schltdl. \& Cham. (a) y Pinus pseudostrobus Lindl. (b).

Los parámetros de ecuaciones, los errores estándar de estimación, los coeficientes de determinación $\left(R^{2}\right)$ y los valores de p se muestran en la tabla 4.

La comparación entre estimaciones de estos modelos, con las ecuaciones encontradas en la literatura (Fig. 6), muestran un buen ajuste $\left(\mathrm{R}^{2}=0.95\right.$ y $\mathrm{R}^{2}=0.93$, para $A$. religiosa y $P$. pseudostrobus, respectivamente) además, no se presentaron diferencias estadísticas significativas entre valores estimados con las ecuaciones halladas en la literatura (Avendaño et al., 2009 y Návar, 2010) y las generadas en el presente estudio, tanto para $A$. religiosa $(\mathrm{P}=0.65)$, como para P. pseudostrobus $(\mathrm{P}=0.90)$. 
TABLA 4. Componentes de los modelos generados para la estimación de biomasa aérea y carbono (kg/árbol) de Abies religiosa (Kunth) Schltdl. \& Cham. y Pinus pseudostrobus Lindl.

\begin{tabular}{|c|c|c|c|c|c|}
\hline Variable & Parámetro & Estimación & Error estándar & t para HO parámetro $=0$ & Prob $>|t|$ \\
\hline A. religiosa & \multicolumn{2}{|c|}{$\mathrm{B}=0.034 \mathrm{DN} 2.7169$} & & & \\
\hline Ordenada al origen & b & 0.0340 & 0.0444 & 0.7650 & 0.4870 \\
\hline DN & k & 2.7169 & 0.3221 & 8.4350 & 0.0011 \\
\hline P. pseudostrobus & \multicolumn{2}{|c|}{$B=0.1549 D^{2.3572}$} & & & \\
\hline Ordenada al origen & $b$ & 0.1549 & 0.2374 & 0.6520 & 0.5498 \\
\hline DN & $\mathrm{k}$ & 2.3572 & 0.3819 & 6.1730 & 0.0035 \\
\hline A. religiosa & \multicolumn{2}{|c|}{$C C=0.01597 D^{2} N^{2.7169}$} & & & \\
\hline Ordenada al origen & b & 0.0160 & 0.0209 & 0.7650 & 0.4870 \\
\hline DN & $\mathrm{k}$ & 2.7169 & 0.3221 & 8.4350 & 0.0011 \\
\hline P. pseudostrobus & \multicolumn{2}{|c|}{$C C=0.07744 \mathrm{DN}^{2.3572}$} & & & \\
\hline Ordenada al origen & $b$ & 0.0774 & 0.1187 & 0.6520 & 0.5498 \\
\hline DN & $\mathrm{k}$ & 2.3572 & 0.3819 & 6.1730 & 0.0035 \\
\hline
\end{tabular}

B = Biomasa aérea; $C C$ = Contenido de carbono; $\mathrm{DN}$ = diámetro normal $(\mathrm{cm}) ; \mathrm{b}, \mathrm{k}$ = parámetros de regresión; $\mathrm{R}^{2}$ = coeficiente de determinación ajustado.

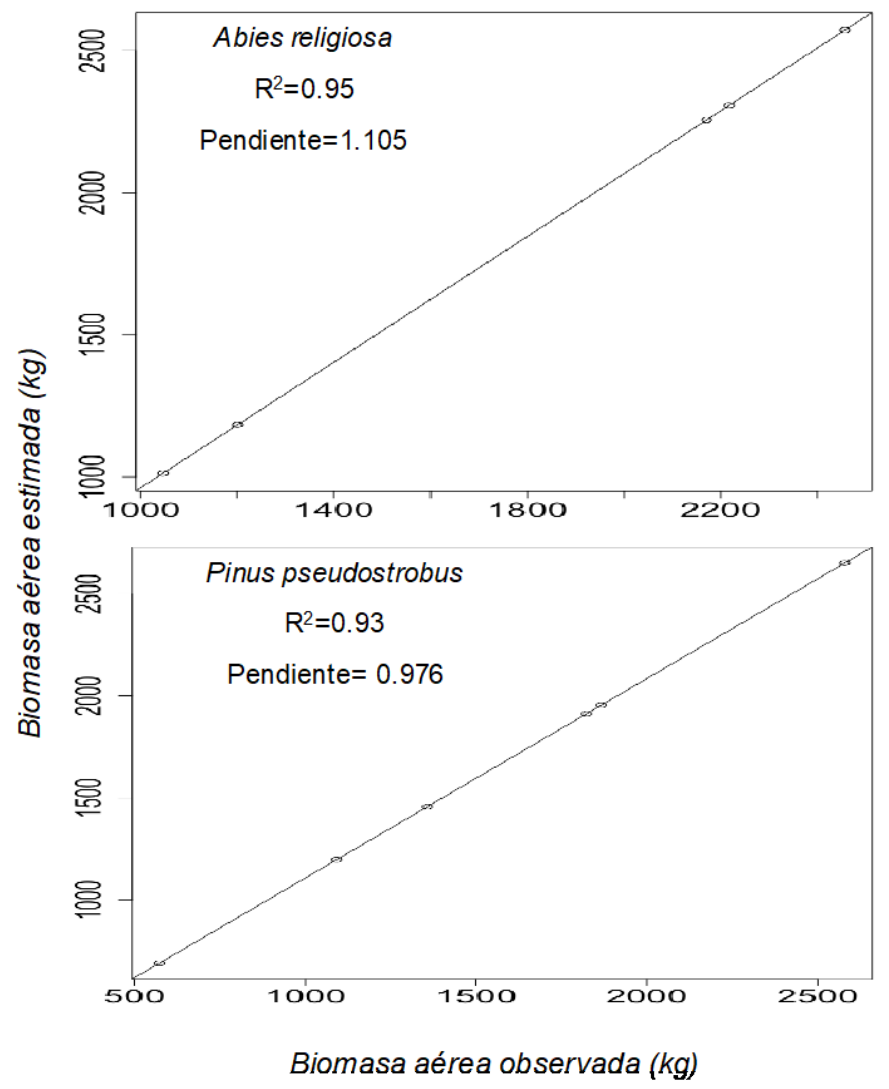

FIGURA 6. Comparación de valores estimados y observados de biomasa aérea (kg) (A. religiosa y P. pseudostrobus). 
La distribución relativa de la biomasa entre los componentes estructurales de los árboles de $A$. religiosa se comportó de la siguiente manera, con promedios de: $82.4 \%$ en el fuste; en ramas de $10.1 \%$; y de $7.5 \%$ en follaje. Para $P$. pseudostrobus la distribución fue la siguiente: promedió $85.4 \%$ en fuste; en ramas $10.0 \%$ y $4.6 \%$ para follaje; similar a lo señalado por López-Escobar et al., (2018) con porcentajes de 89.5, 7.5 y 3 , respectivamente.

\section{Biomasa Aérea (BA)}

La BA en el año 2007 fue de $346.15 \mathrm{Mg} \mathrm{ha}^{-1}$ y $345.25 \mathrm{Mg}$ ha $^{-1}$ para RCM y RSM, respectivamente. Para el 2017 la BA fue 287.62 $\mathrm{Mg} \mathrm{ha}^{-1}$ en el RCM y de $323.23 \mathrm{Mg} \mathrm{ha}^{-1}$, en el RSM. El CC sigue la misma tendencia que la BA, para el año 2007, fue de $166 \mathrm{Mg} \mathrm{ha}^{-1}$ y $165 \mathrm{Mg} \mathrm{ha}^{-1}$ para RCM y RSM, respectivamente.

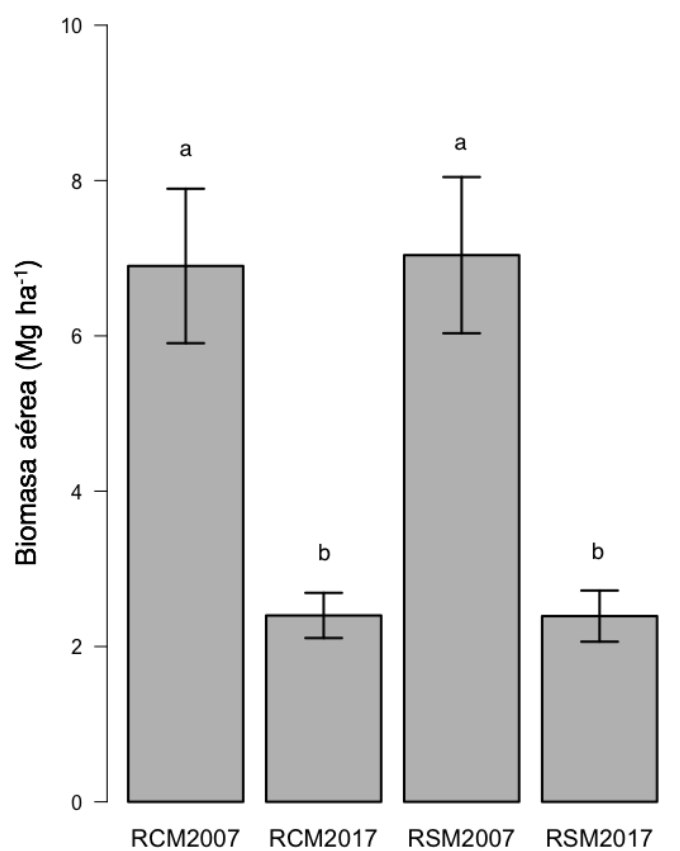

Condición del rodal y año
Para el año 2017, se presentó una acumulación de 119 $\mathrm{Mg} \mathrm{ha}^{-1}$ de carbono para el RCM y de $149 \mathrm{Mg} \mathrm{ha}^{-1}$ en el RSM, no existen diferencias estadísticas significativas entre condición de rodal, solo entre año de muestreo (Fig. 7).

La biomasa aérea y carbono por hectárea presentaron diferencias estadísticas altamente significativas entre rodales y año (Tabla 5). Sin embargo, después de aplicar la prueba Tukey HSD $(\alpha \leq 0.005)$, no existió diferencia estadística significativa entre condición de manejo (RSM y RCM; P $\leq$ 0.99), pero si, entre los años de medición (2007 y $2017 ; \mathrm{P} \leq 0.0001)$ (Fig. 7).

Se puede observar en la figura 7 , que la diferencia estadística significativa, se presenta solo en las medias de distinto año. Demostrado así, que no existen diferencias significativas en biomasa y carbono $\left(\mathrm{Mg} \mathrm{ha}^{-1}\right)$, en rodales con y sin manejo para las mediciones en el mismo año.

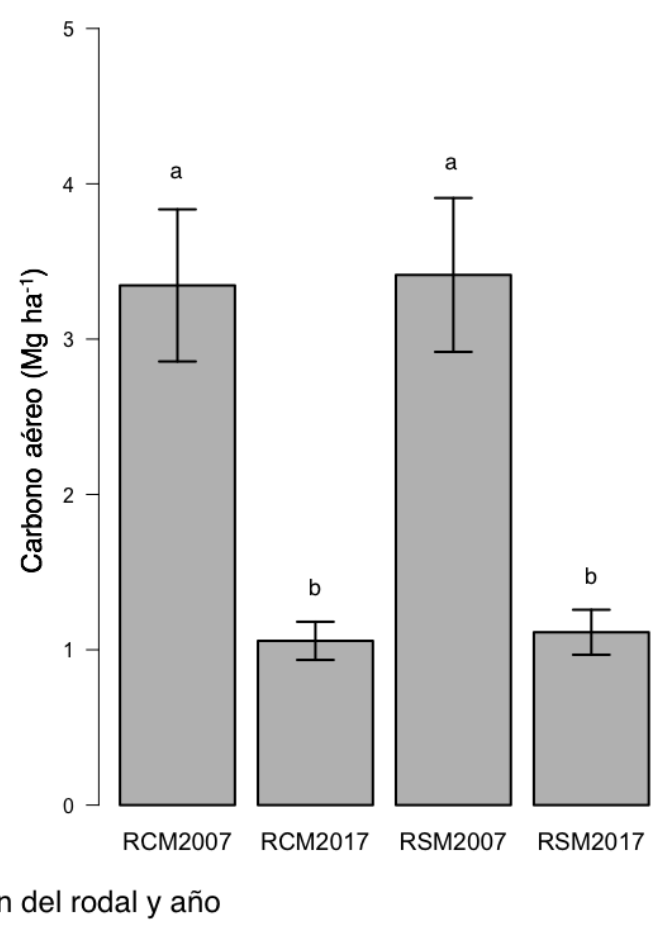

FIGURA 7. Comparación de medias de biomasa aérea y carbono ( $\mathrm{Mg} \mathrm{ha}^{-1}$ por sitio) por condición del rodal (RCM y RSM), para los años 2007 y 2017 (Tukey HSD; $\alpha \leq 0.005$ ). 
TABLA 5. ANOVA para la biomasa aérea y carbono en RSM y RCM (2007 y 2017).

\begin{tabular}{lcccc}
\hline Biomasa & SC & CM & Fo & $\operatorname{Pr}(>F)$ \\
\hline Rodal & 41.04 & 13.682 & 15.81 & $1.21 \mathrm{E}-09$ \\
Residuales & 292.47 & 0.865 & & $1.02 \mathrm{E}-10$ \\
\hline Carbono & & & 17.74 & \\
\hline Rodal & 53.8 & 17.931 & & \\
Residuales & 341.6 & 1.011 & & \\
\hline
\end{tabular}

SC = Suma de cuadrados, $C M=$ Cuadrado de la media, $F_{0}=$ Valor del estadístico F de Fisher y Pr $=$ Probabilidad

\section{DISCUSIÓN}

El sistema silvícola de selección para poblaciones incoetáneas, está diseñado para mantener esta condición, con presencia de individuos en todas las categorías diamétricas, tomando como base la curva de distribución de Liocourt (Smith et al., 1997). En este sentido, la estructura del rodal con manejo se ajusta a la curva irregular indicada, mientras que en el rodal sin manejo existe escasez de individuos en algunas categorías diamétricas (Fig. 3). Lo anterior, debido a la falta de manejo, ya que el desarrollo del rodal está determinado por la corta de regeneración, lo cual promueve la conservación de otros recursos (Smith et al., 1997), además de evitar la acumulación de material combustible en el sotobosque, como efecto de las prácticas forestales, para reducir el riesgo ante un disturbio mayor, permitiendo a los rodales tener una mayor resistencia, robustez y resiliencia, es decir, que mantengan la capacidad para absorber pequeños disturbios y evitar que estos se amplifiquen (antes de modificarse), así como de volver a su estado original (nivel de productividad, composición de especies) después de algún disturbio o, en este caso, del aprovechamiento forestal, protegiendo con esto, biodiversidad, integridad ecológica, procesos de los ecosistemas, potencial productivo, fertilidad del suelo y calidad del agua, por nombrar algunos (Perry et al., 2008). En la etapa de bosque maduro (reiniciación del sotobosque) y al inicio del viejo crecimiento, se tienen los mayores reservorios de carbono (Bormann y Likens, 1979; Oliver, 1981; Fujimori, 2001), considerando los diferentes componentes de los sumideros (IPCC, 2006), y es esta etapa donde se mantienen las masas manejadas con el sistema silvícola de selección, Bormann y Likens (1979) se refirieron a esta etapa como el período en el que la biomasa total (viva y muerta) alcanza su máximo. Oliver (1981) se refirió a esta etapa como bosque maduro o reiniciación del sotobosque, y la definió como el período en el que reaparecen las hierbas, los arbustos. El almacén de la biomasa aérea se mantiene relativamente constante al aplicar sistemas silvícolas semiintensivos, como lo es de selección (Fujimori, 2001). Los bosques con alta tasa de crecimiento son más efectivos para capturar el $\mathrm{CO}_{2}$ de la atmósfera y fijarlo como carbono en sus componentes (Daniel et al., 1982; Perry et al., 2008).

Bajo estos supuestos, y respecto a los resultados del presente estudio, es posible que el rodal con manejo sea más eficiente en los procesos de captura y almacenamiento de carbono en biomasa aérea, ya que más de $55 \%$ de sus individuos se encuentran en las categorías de $10 \mathrm{~cm}$ hasta $30 \mathrm{~cm}$, aunque la cifra se reduce considerablemente en las categorías diamétricas sucesivas, en donde $35 \%$ de sus individuos se encuentran en las categorías entre $35 \mathrm{~cm}$ a 55 $\mathrm{cm}$, y su contenido de carbono representa entre 50\% y $60 \%$ del total de la biomasa aérea. Es decir, por un lado, presenta una densidad tal que, el crecimiento es sostenido y prolongado, con arbolado juvenil, pero además posee individuos de porte grande que concentran en su estructura una mayor cantidad de biomasa aérea (Fujimori, 2001), principalmente en el fuste $(75 \%-85 \%)$. Sin embargo, se debe considerar que en este estudio no se están midiendo los cambios en la biomasa en raíz, ya que el manejo a través 
del tiempo, en algunos casos y bajo ciertas condiciones de productividad de los sitios, puede llevar a una mayor distribución en la biomasa aérea, pero reducir el carbono en compontes subterráneos (Noormets et al., 2015).

Algunos autores como Wang et al. (2000), Ordóñez et al. (2001) y Figueroa-Navarro et al. (2010), han usado los datos de los inventarios forestales para estimar los almacenes de biomasa aérea y carbono, para diferentes especies. En este estudio, después de aplicar las ecuaciones alométricas a los datos de inventario forestal, el rodal con manejo presentó una acumulación de biomasa aérea equivalente a la contenida en el arbolado del rodal sin manejo para el año $2017\left(287.62 \mathrm{Mg} \mathrm{ha}^{-1}\right.$ y de $323.23 \mathrm{Mg}$ $\mathrm{ha}^{-1}$, respectivamente), 15\% menor. Estos resultados son similares y comparables a los valores de biomasa hallados para otros bosques templados mixtos (pino-encino) en el país (Ordóñez et al., 2001; Díaz et al., 2007; RodríguezLaguna et al., 2009; Galicia et al., 2015; Chávez-Aguilar et al., 2016), e incluso al de otros países en ecosistemas similares (Balboa-Murias et al., 2006; García-Gonzalo et al., 2007).

La biomasa aérea y carbono disminuyeron en $20 \%$ de 2007 a 2017 probablemente debido al efecto de la cosecha de las diferentes especies, ya que el rodal con manejo se aprovecha maderablemente desde hace 20 años, es decir, para 2017, el bosque ya había sido cosechado en dos ciclos de corta, a una intensidad de entre $20 \%$ y $30 \%$ del volumen total árbol por hectárea $\left(\mathrm{VTA} h \mathrm{a}^{-1}\right)$, modificando el residual de árboles por hectárea (NAH) y área basal. Estas características dasonómicas determinantes, al ser modificadas mediante el manejo, son indicadoras del establecimiento, la composición, la estructura y el crecimiento del bosque (Daniel et al., 1982; Smith et al., 1997; Schulze et al., 2000). La circunstancia más crítica, en cuanto a crecimiento de los rodales se refiere, es la modificación del número de árboles por hectárea mediante las cortas de regeneración y aclareo, las cuales en el método de selección se realizan al mismo tiempo (Smith et al., 1997), y que permiten mantener a los bosques sanos y productivos, aumentando la eficiencia de las tasas de fotosíntesis y, a su vez, las tasas de almacenamiento de biomasa aérea y carbono, además de la renovación de las masas forestales
(Oliver y Larson, 1990; Smith et al., 1997; Perry et al., 2008). Entre los rodales con y sin manejo las variables anteriormente mencionadas son muy similares en condición y año, a excepción de la densidad para el rodal sin manejo en 2017, que aumentó en $23 \%$ su número de árboles por hectárea, no así en área basal. Lo anterior se explica por la presencia de un disturbio en 2012, presentándose derribo de arbolado sobremaduro por efecto del viento, provocando aperturas del dosel, con mayor regeneración natural, principalmente de latifoliadas, las cuales no representan aumento significativo en área basal y, por lo tanto, tampoco en biomasa aérea. En este contexto, la producción de un rodal (volumen total por árbol o biomasa aérea), se puede modificar mediante el manejo, controlando el número de árboles por hectárea, el área basal residual y, con ello las existencias maderables $\left(\mathrm{m}^{3} \mathrm{ha}^{-1}\right)$ y la propia biomasa aérea $\left(\mathrm{Mg} \mathrm{ha}^{-1}\right)$.

En el sistema silvícola de selección los árboles maduros son removidos de manera individual o en grupos, en periodos relativamente cortos, provocando disturbios de baja intensidad y magnitud, lo cual permite la restructuración original regresando a su estructura y composición inicial (Oliver y Larson, 1990; Perry et al., 2008), ya que los árboles residuales alrededor de las aperturas de la corta de selección proveen semilla y protección necesaria para la regeneración. Este proceso se repite en forma indefinida y con turnos cortos (Fujimori, 2001).

La biomasa aérea de $A$. religiosa y $P$. pseudostrobus (especies con mayor importancia ecológica y económica en la región) mantuvieron la misma tendencia de acumulación que el resto de las especies, aun cuando representan $70 \%$ del volumen cosechado (en 1997 y 2007).

En el rodal con manejo la dominancia relativa de $A$. religiosa es mayor, respecto al rodal sin manejo, siendo la tercera especie en importancia respecto a su IVI (12.3\%), detrás de Quercus laurina y Pinus pseudostrobus, en contraste con el rodal sin manejo, en donde $A$. religiosa, se ubicó en sexta posición con un IVI de 9.3\%. Lo anterior puede explicarse debido a que los árboles en condiciones de competencia, tienden a controlar los espacios de 
crecimiento en el sitio, además de los nutrientes que en este se encuentren (Oliver y Larson, 1990; Perry et al., 2008), y también debido al manejo selectivo, algunas especies como A. religiosa, en ocasiones, prosperan mejor en condiciones de dosel semicerrado o cerrado, a diferencia de las especies latifoliadas en las cuales su regeneración prospera mejor en espacios abiertos. Es por ello que en rodal con manejo las condiciones ambientales que generó el sistema de selección permitieron que las coníferas tengan mayor abundancia de árboles (NAH) y dominancia con mayor área basal dentro del sitio, variables críticas en el IVI de una especie (Curtis y McIntosh, 1951).

Comparando con estudios del mismo tipo (RodríguezLaguna et al., 2009; Galicia et al., 2015), se demuestra que el bosque de la RBMM tiene buena capacidad de recuperación al regresar $80 \%$ su contenido de biomasa aérea, en solo 10 años, al compararse con otros ecosistemas similares, en donde se empleó aproximadamente el doble de tiempo en alcanzar el almacén de biomasa aérea previo a la corta de regeneración (Balboa-Murias et al., 2006; García-Gonzalo et al., 2007; Galicia et al., 2015) y sin contar con la madera extraída producto de dos ciclos de cosecha, que de acuerdo con su historial silvícola se estiman en $1400 \mathrm{~m}^{3}$ VTA (178 $\mathrm{m}^{3}$ ha $^{-1}$ VTA).

Por otro lado, las ecuaciones alométricas de biomasa y carbono, generadas en el presente estudio, demuestran que su uso puede ser confiable en rodales de la RBMM (para el intervalo de diámetros de $20 \mathrm{~cm}$ a $70 \mathrm{~cm}$, aproximadamente), en las mismas condiciones de bosque, y se consideró suficiente usar el DN (m) para la estimación de ambas (Acosta-Mireles et al., 2002; Díaz et al., 2007; Acosta-Mireles et al., 2009; Avendaño et al., 2009; Rodríguez-Laguna et al., 2009), debido a que tanto el modelo original (potencial) como el linealizado (logarítmico) resultaron ser confiables, con base en su bondad de ajuste $\left(\mathrm{R}^{2}=0.9\right)$, además de que no se presentaron diferencias estadísticas significativas ( al compararse con valores estimados de otras ecuaciones encontradas en la literatura para estudios de las mismas características (Avendaño et al., 2009 y Návar, 2010) y las generadas en el presente estudio.
La proporción de la distribución de biomasa entre componentes estructurales se encuentra entre los límites publicados para las mismas especies y otras coníferas en México, e incluso, es similar a la registrada en latifoliadas (Acosta-Mireles et al., 2009; Avendaño et al., 2009; Rodríguez-Laguna et al., 2009; Figueroa-Navarro et al., 2010; Carrillo et al., 2014; Hernández, 2015; López-Escobar 2018). Aunque esta proporción puede variar entre especies y regiones, se hace evidente que esto depende de las condiciones en las que los individuos se desarrollen, como son: tipo de vegetación, manejo, competencia, altitud, latitud, clima, suelo, densidad, entre otras (Oliver y Larson, 1990).

Los efectos de la extracción de biomasa, y su recuperación en bosques manejados, están poco documentados. Al respecto, Figueroa-Navarro et al. (2010) encontraron que en un bosque de Pinus patula Schltdl. et Cham. en Zacualtipán, Hidalgo, rodales manejados registran biomasa arbórea similar a rodales no intervenidos, lo que sugiere que el bosque manejado es eficiente para la captura de carbono atmosférico. Por otro lado, Jandl et al. (2007) concluyeron, en una síntesis relativa a la influencia de las prácticas silvícolas en la captura de carbono, que los bosques manejados con turnos largos aseguran un menor disturbio, o pérdida de carbono. Fujimori (2001) menciona que la mayor acumulación de carbono en el bosque se da en las etapas de desarrollo de bosque maduro, debido a que después de esta comienza un declive en el almacenamiento de este elemento (viejo crecimiento), con bajas tasas de transpiración y crecimiento de las masas, asociado a la baja reincorporación de carbono al suelo en forma de materia orgánica.

La biomasa aérea estimada con las ecuaciones propuestas por Avendaño et al. (2009) y Návar (2010), para Abies religiosa y Pinus pseudostrobus, respectivamente, presentan una tendencia similar a las ecuaciones generadas en el presente estudio, aunque los valores para árboles de mayor diámetro (los que más influyen en las estimaciones de biomasa aérea), se tienden a sobreestimar, aunque cabe señalar que esta diferencia no es significativa $(\mathrm{P} \leq 0.65)$. De lo más destacable es que el método de selección bien 
aplicado puede mantener la estructura y composición de los rodales, tanto para la captura de carbono, como para que la mariposa monarca pueda hibernar.

\section{CONCLUSIONES}

Los rodales con y sin manejo, almacenan una cantidad equivalente de biomasa aérea y carbono por hectárea, además de que mantienen condiciones similares de estructura y composición.

El almacenamiento de biomasa aérea en el rodal con manejo, respecto al ciclo de corta anterior (2007), y después de dos cortas de regeneración (desde hace 20 años), disminuyó en $20 \%$; sin embargo, se mantuvo similar a lo largo de dos ciclos de corta, no se encontraron diferencias significativas en acumulación, con respecto al rodal sin manejo. Por lo tanto, los rodales con manejo forestal de los bosques de pino-encino en la RBMM, podrían alcanzar valores de acumulación de biomasa aérea y carbono, similares a los de rodales sin manejo, o conservados en su estado natural, en un menor tiempo.

Las ecuaciones de biomasa aérea y carbono resultaron ser confiables, y pueden ser utilizadas en rodales de la RBMM de las mismas condiciones de bosque del estudio.

La mayor asignación de biomasa aérea en el rodal con manejo para las coníferas, se presenta en el fuste (84\%), seguido de las ramas $(10 \%)$ y follaje $(6 \%)$, lo cual indica que el manejo de selección, mediante el control de la densidad (número de árboles ha-1 y área basal), favorece la acumulación de biomasa y carbono hacia este componente, además de ser el principal producto maderable, aumentando la calidad de la madera (en teoría, al tener menos ramas) y la eficiencia de captura de carbono, principales objetivos de los bosques bajo aprovechamiento maderable.

El manejo forestal a través el sistema silvícola de selección mantiene la estructura y composición, condiciones ambientales críticas en el rodal, que permiten almacenar una cantidad de biomasa similar a la de un bosque sin manejo, por lo tanto, los bosques manejados se pueden considerar sumideros eficientes de carbono, sin sumar el contenido de este elemento de la madera extraída producto del aprovechamiento, para lo cual sería necesario realizar futuras investigaciones, para demostrarlo de manera cuantitativa.

\section{REFERENCIAS}

Acosta-Mireles, M., Vargas-Hernández, J., Velázquez-Martínez, A. \& Etchevers-Barra, J. D. (2002). Estimación de la biomasa aérea mediante el uso de relaciones alométricas en seis especies arbóreas en Oaxaca, México. Agrociencia, 36(6), 725-736.

Acosta-Mireles, M., Carrillo-Anzures, F., \& Díaz-Lavariega, M. (2009). Determinación del carbono total en bosques mixtos de Pinus patula Schl. et Cham. Terra Latinoamericana, 27(2), 105-114.

Acosta, M., Carrillo, F., \& Gómez, R. (2011). Estimación de biomasa y carbono en dos especies de bosque mesófilo de montaña. Revista Mexicana de Ciencias Agricolas, 2, 529-543.

Aguilar, R., Ghilardi, A., Vega, E., Skutsch, M., \& Oyama, K. (2012). Sprouting productivity and allometric relationships of two oak species managed for traditional charcoal making in central Mexico. Biomass and Bioenergy, 36, 192-207.

Aguilar-Hernández, L., García-Martínez, R., Gómez-Miraflor, A. \& Martínez-Gómez, O. (2016). Estimación de biomasa mediante la generación de una ecuación alométrica para madroño (Arbutus xalapensis). IV Congreso Internacional y XVIII Congreso Nacional de Ciencias Agronómicas. Universidad Autónoma Chapingo.

Avendaño, D. M., Acosta, M., Carrillo, F., \& Etchevers, J. D. (2009). Estimación de biomasa y carbono en un bosque de Abies religiosa. Revista Fitotecnia Mexicana, 32(3), 233-238.

Balboa-Murias, M. A., Rodríguez-Soalleiro, R., Merino, A., \& ÁlvarezGonzález, J. G. (2006). Temporal variations and distribution of carbon stocks in aboveground biomass of radiata pine and maritime pine pure stands under different silvicultural alternatives. Forest Ecology and Management, 237, 29-38.

Comisión Nacional de Áreas Naturales Protegidas [Conanp]. (2001). Programa de manejo de la reserva de la biosfera Mariposa Monarca. Recuperado de http://www.conanp.gob.mx/que_ hacemos/pdf/programas_manejo/monarca.pdf.

Carrillo, F., Acosta, M., Flores, E., Juárez, J. E., \& Bonilla, E. (2014). Estimación de biomasa y carbono en dos especies arbóreas en La Sierra Nevada, México. Revista Mexicana de Ciencias Agrícolas, 5(5), 779-793.

Chávez-Aguilar, G., Ángeles-Pérez, G., Pérez-Suárez, M., López-López, M. A., García-Moya, E. \& Wayson, C. (2016). Distribución de biomasa aérea en un bosque de Pinus patula bajo gestión forestal 
en Zacualtipán, Hidalgo, México. Madera y Bosques, 22(3), 23-36. doi: $10.21829 /$ myb.2016.2231454

Correa, J. C., Iral, R., \& Rojas, L. (2006). Estudio de potencia de pruebas de homogeneidad de varianza. Revista Colombiana de Estadística, 29, 57-76.

Curtis, J. T. \& McIntosh, R. P. (1951). An upland forest continuum in the prairie-forest border region of Wisconsin. Ecology, 32(3), 476496.

Daniel, T. W., Helms, J. A., \& Baker, F. S. (1982). Principios de silvicultura (1a. ed. en español). México: McGraw-Hill.

Díaz, F. R., Acosta, M., Carrillo, F., Buendía, E., Flores, E., \& Etchevers, J. D. (2007). Determinación de ecuaciones alométricas para estimar biomasa y carbono en Pinus patula Schl. et Cham. Madera y Bosques, 13(1), 25-34. doi: 10.21829/myb.2007.1311233

Díaz-Ríos, M. J., Vázquez-Alarcón, A., Uribe-Gómez, M., SánchezVélez, A., Lara-Bueno, A., \& Cruz-León, A. (2016). Ecuaciones alométricas para estimar biomasa y carbono en aile obtenidas mediante un método no destructivo. Revista Mexicana de Ciencias Agricolas, 16, 3235-3249.

Dirzo, R. (2001). Forest ecosystems functioning, threats and value: Mexico as a case study. In: Managing Human-Dominated Ecosystems. Monographs in Systematic Botany, 84. Missouri Botanical Garden Press. St Louis Missouri.

Figueroa-Navarro, C. M., Ángeles-Pérez, G., Velázquez-Martínez, A., \& De los Santos-Posadas, H. M. (2010). Estimación de la biomasa en un bosque bajo manejo de Pinus patula Schltdl. et Cham. en Zacualtipán, Hidalgo. Revista Mexicana de Ciencias Forestales, 1(1), 105-112.

Fujimori, T. (2001). Ecological and silvicultural strategies for sustainable forest management. Tokyo, Japon: Elsevier.

Galicia, L., Saynes, V., \& Campo, J. (2015). Biomasa aérea, biomasa subterránea y necromasa en una cronosecuencia de bosques templados con aprovechamiento forestal. Botanical Sciences, 93(3), 473-484.

García, E. (2004). Modificaciones al sistema de clasificación climática Köppen ( $5^{a}$ ed). Serie Libros. No. 6. México: Instituto de Geografía, UNAM.

García-Gonzalo, J., Peltola, H., Briceño-Elizondo, E., \& Kellomaki, S . (2007). Changed thinning regimes may increase carbon stock under climate change: A case study from a Finnishboreal forest. Climatic Change, 81, 431-454.

González de Tanago, J., Lau, A., Bartholomeus, H., Herold, M., Avitabile, V., Raumonen, P., Martius, Ch., Goodman, R. C., Disney, M., Manuri, S., Burt, A., \& Calders, K. (2017). Estimation of above-ground biomass of large tropical trees with terrestrial LiDAR. Methods in Ecology and Evolution, 0, 1-12.

Hernández, O. E. (2015). Estimación de los almacenes de carbono en bosque templado, caso de estudio predio particular "CEBATI", municipio de San José del Rincón, Estado de México. Tesis. Universidad Autónoma del Estado de México, Toluca, Estado de México.

Hurlbert, S. H. (1984). Pseudoreplication and the design of ecological field experiments. Ecological Monographs, 54(2), 187-211.

Husch, B. (2001). Estimación del contenido de carbono de los bosques. In: Simposio internacional, medición y monitoreo de la captura de carbono en ecosistemas forestales. Valdivia, Chile.

Instituto Nacional de Estadística y Geografía [Inegi] (2014). Conjunto de datos vectoriales Perfiles de suelos. Escala 1:1,000,000. Instituto Nacional de Estadística, Geografía e Informática, Aguascalientes, México.

Instituto Nacional de Estadística y Geografía [Inegi] (2016). Conjunto de Datos Vectoriales de Uso del Suelo y Vegetación Serie III. Instituto Nacional de Estadística, Geografía e Informática, Aguascalientes, México.

Intergovernmental Panel on Climate Change [IPCC] (2001). Third assessment report-climate change, The scientific basis: summary for policymakers. A report of working group I of the Intergovernmental Panel on Climate Change. UNEP-WMO. P. 7.

Intergovernmental Panel on Climate Change [IPCC] (2006). Guidelines for national greenhouse gas inventories. Eggleston, H. S., L. Buendia, K. Miwa, T. Ngara \& K. Tanabe (eds.). Institute for Global Enviromental Strategies (IGES). Hayama, Japan

Jandl, R., Linder, M., Vesterdal, L., Bauwens, B., Baritz, R., Hagedorn, F., Johnson, D. W., Minkkinen, K., \& Byrne, K. A. (2007). How strongly can forest management influence soil carbon sequestration? Geoderma, 137(3-4), 253-268.

Jiménez, J., Treviño, E. J., \& Yerena, J. I. (2013). Concentración de carbono en especies del bosque de pino-encino en la Sierra Madre Oriental. Revista Mexicana de Ciencias Forestales, 4(17), 50-61.

Kollman, F. (1959). Tecnología de la madera y sus aplicaciones. Madrid, España: Springer Verlag.

Little, T. M. \& Jackson, F. (1976). Métodos Estadísticos para la Investigación en la Agricultura. México: Trillas.

López-Escobar, N. F., Gómez-Guerrero, A., Velázquez-Martínez, A., Fierros-González, A. M., Castruita-Esparza, L. U., \& VeraCastillo, J. A. G. (2018). Reservorios y dinámica de nutrientes en dos rodales bajo aprovechamiento de Pinus montezumae Lamb. en 
Tlaxcala, México. Revista Chapingo Serie Ciencias Forestales y del Ambiente, 24(1), 115-129. doi: 10.5154/r.rchscfa.2017.09.055

MacDicken, K. G. (1997). A guide to monitoring carbon storage in forestry and agroforestry projects. Arlington, VA: Winrock International Institute for Agricultural Development.

NASA. (2018). Mauna Loa Observatory, Hawaii. Administración Nacional de la Aeronáutica y del Espacio (NOAA). Cambio Climático Global. Recuperado de https://climate.nasa.gov/vital-

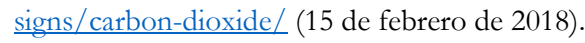

Návar, J. (2010). Alometría para biomasa en especies arbóreas del noroeste de México. Tropical and subtropical agroecosystems, 12, 507519.

Noormets A., Epron, D., Domec, J. C., McNulty, S.G., Fox, T., Sun, G., King, J. S. (2015). Effects of forest management on productivity and carbon sequestration: A review and hypothesis. Forest Ecology and Management, 355(1), 124-140.

Oliver, C. D. \& Larson, B. C. (1990). Forest stand dynamics. New York: Macgraw-Hill.

Ordóñez, J., De Jong, B., \& Masera, O. (2001). Almacenamiento de carbono en un bosque de Pinus pseudostrobus en Nuevo San Juan, Michoacán. Madera y Bosques, 7(2), 27-47. doi: $10.21829 /$ myb.2001.721310

Picard, N., Saint-André, L., \& Henry, M. (2012). Manual de construcción de ecuaciones alométricas para estimar el volumen y la biomasa de los árboles: del trabajo de campo a la predicción. FAO-Centre de Coopération Internationale en Recherche Agronomique pour le Développement, Rome, Montpellier.

Perry, D. A., Oren, R., \& Hart, S. C. (2008). Forest ecosystems (2nd ed). Baltimore. MD: Johns Hopkins University Press.

Rackley, S. A. (2010). Carbon capture and storage. Burlington, MA, USA: Elsevier.

Rodríguez-Laguna, R., Jiménez-Pérez, J., Aguirre-Calderón, O. A., Treviño-Garza, E. J., \& Razo-Zárate, R. (2009). Estimación de carbono almacenado en el bosque de pino-encino en la Reserva de la Biosfera El Cielo, Tamaulipas, México. Ra Ximhai, 5(3), $317-$ 327.

Rojas-García, F., De Jong, B., Martínez-Zurimendí, P., \& Paz-Pellat, F. (2015). Database of 478 allometric equations to estimate biomass for Mexican trees and forests. Annals of Forest Science, 72, 835-864.

Rojas-García, F. y Villers-Ruíz, L. (2008). Estimación de la biomasa forestal del Parque Nacional Malinche: Tlaxcala-Puebla. Revista Ciencia Forestal en México, 33(104), 59-86.
Ruiz-Aquino, F., Valdez-Hernández, J. I., Manzano-Méndez, F., Rodríguez-Ortiz, G., Romero-Manzanares, A., \& Fuentes-López, M. E. (2014). Ecuaciones de biomasa aérea para Quercus laurina y Q. crassifolia en Oaxaca. Madera y Bosques, 20(2):33-48. doi: 10.21829/myb.2014.202162

Schulze, E. D., Wirth, Ch., \& Heimann, M. (2000). Managing forests after Kyoto. Science, 289(5487), 2058-2059. doi: 10.1126/science.289.5487.2058

Shapiro, S. S. \& Wilk, M. B. (1965). An analysis of variance test for normality (Complete Samples). Biometrika, 52(3/4), 591-611.

Slifker, J. F. \& Shapiro, S. S. (1980). The Johnson System: selection and parameter estimation. Technometrics, 22(2), 239-246.

Smith, D. M., Larson, B. C., Kelty, M. J., \& Ashton, P. M. S. (1997). The practice of silviculture: applied forest ecology (9th ed). New York: John Wiley \& Sons.

Ter-Mikaelian, M. T., \& Korzukhin, M. D. (1997). Biomass equation for sixty-five North American tree species. Forest Ecology and Management, 97, 1-24.

Wang, R., Letchford, T., Corneau, P., \& Kimmins, J. (2000). Above- and below-ground biomass and nutrient distribution of a paper birch and subalpine fir mixed-species stand in the sub-boreal spruce zone of British Columbia. Forest Ecology and Management, 130, 1726.

WRB. (2015). Base referencial mundial del recurso suelo 2014, Actualización 2015. Sistema internacional de clasificación de suelos para la nomenclatura de suelos y la creación de leyendas de mapas de suelos. Informes sobre recursos mundiales de suelos 106. Roma: FAO.

Manuscrito recibido el 16 de junio de 2018

Aceptado el 8 de mayo de 2019

Publicado el 17 de marzo de 2020

Este documento se debe citar como:

Hernández-Moreno, J. A., Velázquez-Martínez, A., FierrosGonzález, A. M., Gómez-Guerrero, A., Reyes-Hernández, V. J., \& Gil Vera-Castillo, J. A. (2020). Estimación de biomasa aérea y carbono, en rodales con y sin manejo forestal en la Reserva de la Biosfera Mariposa Monarca. Madera y Bosques, 26(1), e2611802. doi: 1021829/myb.2020.2611802

Madera y Bosques por Instituto de Ecología, A.C. se distribuye bajo una Licencia Creative Commons Atribución-NoComercialCompartirlgual 4.0 Internacional. 